\title{
The stellar parameters of the SMC WR binary HD 5980
}

\author{
Jörg Schweickhardt ${ }^{1}$ and Werner Schmutz ${ }^{2}$ \\ ${ }^{1}$ Landessternwarte Königstuhl, D-69117 Heidelberg, Germany \\ ${ }^{2}$ Institut für Astronomie, ETH-Zentrum, CH-8092 Zürich, Switzerland
}

\begin{abstract}
We present the temporal variation of the stellar parameters of the erupting star in HD 5980. We derive the effective temperature and radius from the observed changes of the visual magnitude. Our analysis is based on the assumption that the luminosity of the erupting star remained constant and that the brightness of the second star did not vary.
\end{abstract}

\section{The determination of the stellar parameters $T_{*}$ and $R_{*}$}

A simple method to derive the changes of the parameters $T_{*}$ and $R_{*}$ of a star is to analyze the variations of the visual magnitude $\mathrm{M}_{\mathrm{v}}$. Using Stefan-Boltzmann's law and the dependence of the observed flux, $f_{\lambda}$, on distance one gets

$$
\sigma T_{*}^{4}=\pi F_{\lambda} \frac{\int_{0}^{\infty} f_{\lambda} d \lambda}{f_{\lambda}}
$$

Knowing the luminosity $L \propto \int_{0}^{\infty} f_{\lambda} d \lambda$ and the visual magnitude $\mathrm{M}_{\mathrm{v}} \propto \log f_{\lambda}$, $\lambda=5555 \AA$, only the theoretical knowledge of the astrophysical flux $F_{\lambda}$ emitted in the $\mathrm{V}$ band is needed to derive the temperature and the radius with equation (1). As a simple approximation we use here the Planck function $B_{\lambda}\left(T_{*}\right)$ for $\mathrm{F}_{\lambda}$.

If we want to apply this method to the binary system HD 5980, we need additional assumptions: (a) the LBV-like outburst in 1994 is the result of the eruption of only one star (star A, classified as an O7I star before eruption); (b) the luminosity of the erupting star remains constant with time; and (c) there is no variability of the companion (star $\mathrm{B}, \mathrm{WN} 4$ ). With the luminosity $L=3 \times 10^{6} \mathrm{~L}_{\odot}$ and the constant $\mathrm{M}_{v}$ (star $\left.\mathrm{B}\right)=-6.3 \mathrm{mag}$ from Koenigsberger et al. (1998), we derive a temperature of $31135 \mathrm{~K}$ and a radius of $60 \mathrm{R}_{\odot}$ for Dec. 30,1994 . These values are slightly different from Koenigsberger et al. (1998) $\left(T_{*}=35500 \mathrm{~K}, R_{*}=48 \mathrm{R}_{\odot}\right)$ as expected. With the approach $F_{\lambda}=B_{\lambda}(T)$, we get the approximate temperature and radius of the photosphere $(\tau \simeq 1)$, whereas Koenigsberger et al. (1998) calculate these parameters for an inner border of a non-LTE model atmosphere at $\tau_{\text {Ross }} \simeq 20$.

\section{HD 5980 from 1987 till 1997: $M_{V}$ from Albert Jones}

HD 5980 has been observed by Albert Jones visually over more than ten years (Jones \& Sterken 1997). A careful inspection of the published visual magnitude of HD 5980 reveals a systematic difference between photometric observations and visual brightness estimates. We find that Jones' visual magnitudes have to be adjusted by $+0^{\mathrm{m}} 6$ to put them on the $V$ scale. In Fig. 1 (left) the resulting 

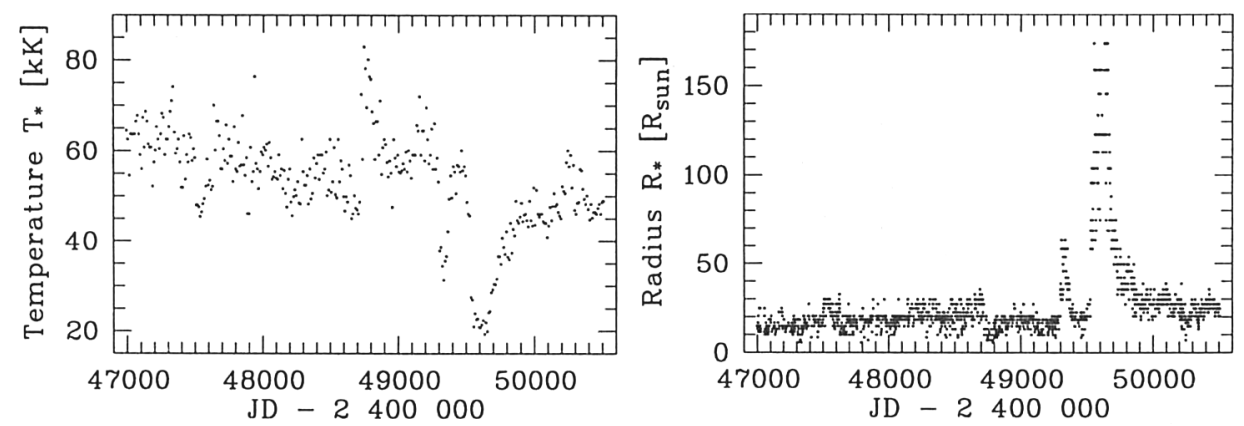

Figure 1. Variation of the effective temperature (left) and the radius (right) of HD 5980's erupting star between 1987 and 1997.

effective temperature of the eruptive star in HD 5980 is shown as a function of time. Adopting the luminosity given above, this temperature variation can also be converted to a variation of the stellar radius, which is illustrated in Fig. 1 (right). The two figures demonstrate the large variations of the stellar parameters of the eruptive star.

\section{Observations in 1995: microvariations}

A photometric monitoring campaign of Sterken \& Breysacher (1997) in Nov/Dec 1995 (16 months after the outburst) revealed a $6-\mathrm{hr}$ periodic oscillation that was visible in the Strömgren $b$ and $y$ band. The most straightforward explanation of the nature of this short-period variability is radial pulsations.

Using the same method as above, we can derive temperature and radius variations from these photometric oscillations (Fig. 2).
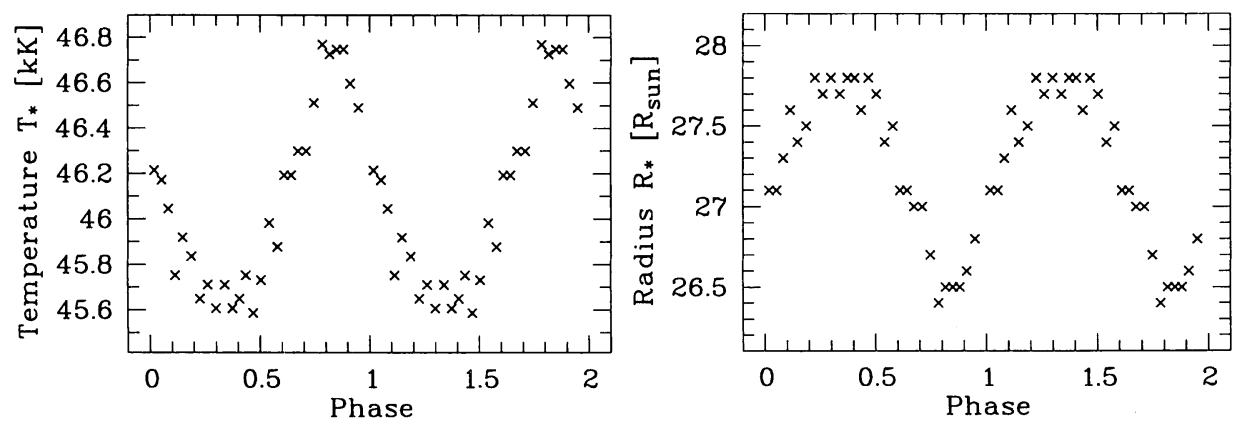

Figure 2. Variation of the effective temperature (left) and the radius (right) of HD 5980's eruptive star within 6 hours in Nov/Dec 1995. The epoch is arbitrary.

\section{References}

Jones, A., Sterken, C. 1997, J. Astron. Data 3, 4

Koenigsberger, G., Peña, M., Schmutz, W., Ayala, S. 1998, ApJ 499, 889

Sterken, C., Breysacher, J. 1997, A\&A 328, 269 